\title{
Flora and vegetation of the different habitats of the western Mediterranean region of Egypt.
}

\author{
K. H. Shaltout ${ }^{1}$, H. A. Hosni ${ }^{2}$, R. A. El-Fahar ${ }^{1}$ and D. A. Ahmed ${ }^{1}$ \\ ${ }^{1}$ Botany Department, Faculty of Science, Tanta University, Tanta, Egypt \\ ${ }^{2}$ Botany Department, Faculty of Science, Cairo University, Cairo, Egypt. \\ E-mail addresses: kamal.shaltout@ @ science.tanta.edu.eg, \\ h.hosni@yahoo.com, raelfahar@yahoo.com, \\ dalia.ahmed@ science.tanta.edu.eg*, drdalia1080@yahoo.com.
}

K. H. Shaltout, H. A. Hosni, R. A. El-Fahar and D. A. Ahmed, 2015. Flora and vegetation of the different habitats of the western Mediterranean region of Egypt. Taeckholmia 35: 4576.

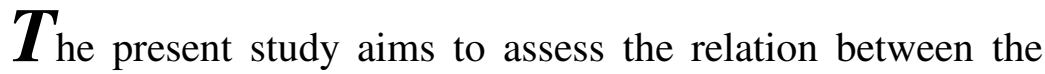
floristic composition and the different habitats charcterizing the western Mediterranean region of Egypt, determine the community types that dominate the study area; and evaluating the rarity of the recorded species. Nine hundred and sixty eight species (belonged to 429 genera and 97 family) were recorded; 363 species were restricted to one habitat, while only one species occured in all the 13 habitats. Asteraceae had the highest contribution, followed by Fabaceae, Poaceae and Brassicaceae. The most represented life form was therophytes, while parasites were the least. Six habitat groups were resulted after the application of TWINSPAN and DCA. Mediterranean elements were the most represented, followed by Irano-Turanian, but SudanoZambezian elements were the least. Twenty endemics and 21 near endemic species were recorded. Species which had small geographical distribution, narrow habitat specificity and were non-abundant (SNN) were the most represented, while species had large geographical distribution, wide habitat specificity and non-abundant (LWN) were the least. Forty one species

Received 18 August, Accepted 2 October 2015 
were recorded as introduced species in the study area. The recent land use led to the emergence of new invasive species, which may severely affect the plant diversity and community structure of the study area.

Key words: Egypt, habitat, flora, vegetation, western Mediterranean.

\section{Introduction}

The North-Western Mediterranean Desert of Egypt is diversified with the coastal area, wadis, depressions and terrain of varying degrees of accessibility. The area encloses different water sources and varied irrigation schemes. Rainwater is the basis of life and economic activity in the coastal territories, with only moderate contributions from underground water. The range of economic activities is substantial, covering rainfed agriculture, livestock rising, trade, tourism, industry, mining, quarries, petroleum and various services (Batanouny, 1999). The major studies which deal with the entire flora of Egypt, or partly in their context, were those of Forsskål, Delile, Ascherson and Schweinfurth, Muschler, and Ramis (see Täckholm and Drar 1941, 1950, 1954, 1969), Montasir and Hassib (1956) Täckholm (1956, 1974), El-Hadidi (1980, 1993, 2000), Cope and Hosni (1991), Boulos and El-Hadidi (1994), El-Hadidi and Fayed (1994/95) and Boulos (1999, 2000, 2002, 2005, 2009). These studies include valuable, but scattered information about the flora of the North-Western Mediterranean region of Egypt. The only study that has a full list of the flora of this region is that of Hassib (1951) and Ahmed (2009).

Many phytosociological studies were carried in the study area. Ammar (1970) recorded 107 species in the rocky ridges at Burg El-Arab, Hilmy (1971) studied the distribution of Asphodelus microcarpus and associated species along the western Mediterranean coastal land, El-Ghareeb (1975) recorded 89 species in the saline and marshy habitats, El-Bayyoumy (1976) studied the sand dune vegetation at Mariut area, El-Kady (1980) Studied the effect of grazing pressures and some other ecological parameters on some fodder plants, Shaltout (1983) studied the distribtiuon of Thymelaea hirsuta and associated species, El-Kady (1987) recorded 132 species in Maktala, Kamal (1988) recorded 230 species in the Western Mediterranean Desert, Heneidy (1991) studied the grazing systems of Mariut, while Fakhry (1994) recorded 316 species in the study area. 
The western Mediterranean coastal region of Egypt can be distinguished into two main provinces: an eastern province between Alexandria and Ras El-Hekma, and a western province between Ras El-Hekma and Sallum (Fig. 1). The landscape of this region was distinguished into a northern coastal plain and a southern tableland (Selim, 1969). Generally, the eastern province can be subdivided into two distinct physiographic areas, each with its own particular topographical features (FAO, 1970): the area from Alexandria to Alamein, which includes three main ridges running parallel to the coast and flat depressions in between, and the area from Alamein to Ras El-Hekma, which consists of an irregular succession of alternating low hills and

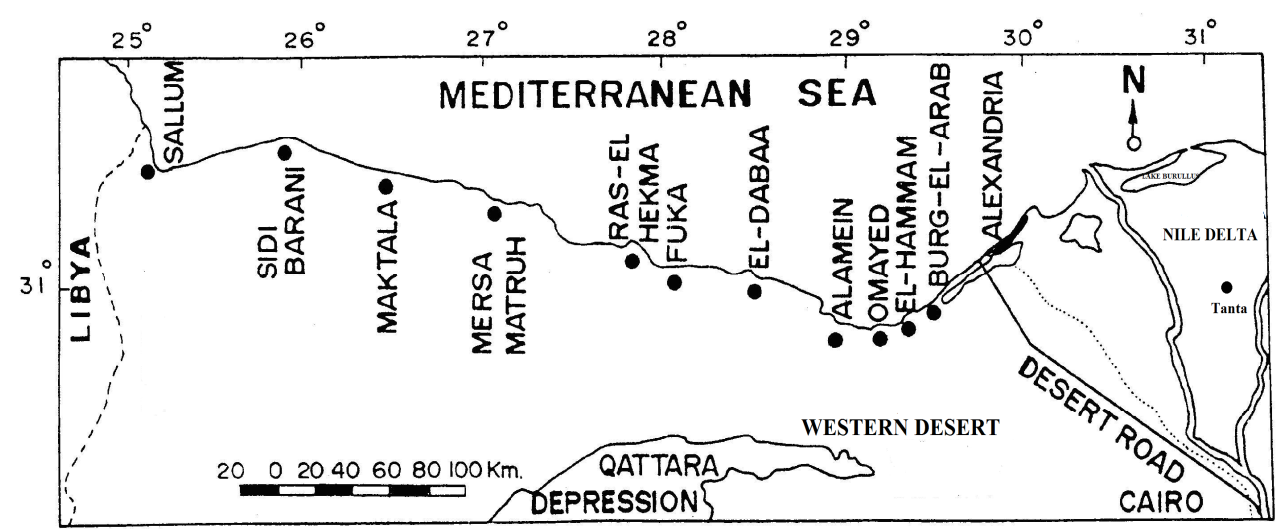

Fig. 1. Map of the study sites $(\bullet)$ in the Western Mediterranean Desert of Egypt.

closed depressions, sloping from south to north. There is an almost continuous range of dunes along the coast. Thirteen major habitats were identified in this region: sand dunes, sand formations, saline depression, salt marshes, non-saline depression, inland ridges, inland plateau, wadis, cultivated land (include rainfed and irrigated farms), road sides, summer resorts, Sallum plateau and Lake Mariut (Ahmed, 2009).

The prevailing climate can be qualified as an arid Mediterranean with mild winters (UNESCO, 1977). January is the coldest month, while August is the hottest. The lowest mean minimum air temperature varies between $7.3{ }^{\circ} \mathrm{C}$ at El-Dabaa in January and $23.5{ }^{\circ} \mathrm{C}$ at Dekheila in August. The highest mean maximum air temperature varies between $17.1^{\circ} \mathrm{C}$ at Ras ElHekma in January and $30.6{ }^{\circ} \mathrm{C}$ at Alexandria in August. The relative humidity varies between $54 \%$ at Sallum in March and $80 \%$ at Ras ElHekma in June and July. In winter months, the evaporation varies between 
$3.8 \mathrm{~mm} \mathrm{day}^{-1}$ in December at Alexandria and $7.3 \mathrm{~mm} /$ day in February at Mersa Matruh. In summer months, the records vary between $5.6 \mathrm{~mm} /$ day in July at Alexandria and $9.7 \mathrm{~mm}_{\text {day }}{ }^{-1}$ in June at Sallum.

The distribution of the mean annual rainfall shows that the amount of annual rainfall decreases sharply from about $150 \mathrm{~mm}$ near the coast to 80 $\mathrm{mm}$ at a distance of $160 \mathrm{~km}$ inland. Most of the rain falls during winter (60\% or more from November to February), and the summer is virtually dry. Wind in this region is generally strong and violent; dust storms and pillars were not rare. Dry hot dust-laden winds from the south known as Khamasin blow occasionally for about 50 days during spring and early summer. During winter and early spring, winds blow strongly with an average velocity of about 20-23 km hour ${ }^{-1}$. Wind speed decreases in May and June, but July is windy. The end of summer is characterized by many calm days and the average wind speed drops to $15 \mathrm{~km} \mathrm{hour}^{-1}$ (Shaltout, 1983).

The main land uses in the study area were grazing and rainfed farming (or irrigated by underground and runoff water). The main annual crop is barley (Hordeum vulgare L.). Irrigated agriculture of pasture and grain crops and fruit trees (mainly vines) is spreading after the extension of irrigation canals from the Nile up to $60 \mathrm{~km}$ west of Alexandria (Zahran and Willis, 2009). Vegetables such as tomatoes, onions, broad beans and watermelons were cultivated mostly in small plots for local consumption. Sheep, goats, camels, donkeys and cattle were found in the study area, but animal production is concerned mainly with sheep and goats. The goats were used largely to supply the Bedouins with meat and milk (Seif El-Nasr and Bidak, 2005). The observed land use changes from a structure based on pastoralism to a structure based on multiple uses or on a group of economic activities in which agriculture has an increasing role (El-Kady et al. 1995). Oil and gas fields with their shipping facilities, and gypsum extraction were among land use activities (Kassas, 1979). Nowadays, summer resorts and tourist sites occupy the shoreline zone from Burg El-Arab to Mersa Matruh (El- Fahar and Sheded, 2002).

The present study aims to assess the relation between the floristic composition and the different habitats charcterizing the western Mediterranean region of Egypt, determine the community types that dominate the study area; and evaluating the rarity of the recorded species. 


\section{Material and methods}

Forty one field trips were conducted seasonally to cover different locations in Western Mediterranean coast during three years from spring 2004 to spring 2007. The main habitats and community types prevailing in each location were determined through visiting different sites. Specimens of the recorded species were collected from the different sites of each location; for the purpose of revealing the natural complexity of the floristic elements in the western Mediterranean region. Floristic records were carried out, based on the presence/absence of species, taking into consideration the type of habitats and plant associations. The plant identification were carried out by consulting the following references: Täckholm (1974), the specimens were matched in Cairo University Herbarium (CAI) and the name updated according to Boulos (2009), all the collected Herbarium speciemens were deposited in Tanta Herbarium (TANE). Life forms of the recorded species were identified following the system of Raunkiaer (1937).

Global geographical distributions of the recorded species were gathered from Tutin et al. (1964, 1968, 1972, 1976), Zohary (1966, 1972), Wickens (1976), Meikle (1977, 1985), Feinbrun-Dothan (1978, 1986), Chitek and Slavik (1993), Snogerup and Snogerup (1993), Boulos (1999, 2000, 2002, 2005), Abdel-Khalik (2002), Shaltout and Al-Sodany (2002) and Seif ElNasr and Bidak (2005). The Endemic and near endemic species in the study area were recorded acoording to Boulos (2009), and the introduced species were recorded according to Täckholm (1956 and 1974) and Boulos (1999, 2000, 2002 and 2005). Rarity forms of the recorded species were assessed depending on the national geographical range, habitat specificity and local abundance according to scheme of Rabinowitz (1981): large geography, wide habitat and abundant species (LWA); large geography, wide habitats and non-abundant species (LWN); large geography, narrow habitats and abundant species (LNA); large geography, narrow habitats and non-abundant species (LNN); small geography, wide habitats and abundant species (SWA); small geography, wide habitats and non-abundant species (SWN); small geography, narrow habitats and abundant species (SNA) and small geography, narrow habitats and non-abundant species (SNN). The weeds, which identified in the study area, were divided into ruderal and segetal weeds according to habitat in which they were recorded.

Two trends of multivariate analysis were applied in the present study: twoway indicator species analysis (TWINSPAN), as a classification technique 
(Hill 1979a), and detrended correspondence analysis (DCA), as an ordination one (Hill 1979b). Linear correlation coefficient was applied to find out the relationships between number of species, genera and families and number of habitats in which they occur (SPSS 2006).

\section{Results}

The relation between the number of species, genera and families on one side and number of habitats in which they occur on the other side indicated a negative relationship between them (Fig. 2). Nine hundred and sixty eight species were recorded in the study area belonging to 429 genera and 97 families. Asteraceae had the highest contribution (126 species $=13.0 \%$ of the total), followed by Fabaceae $(114=11.8 \%)$, Poaceae $(119=12.3 \%)$, Brassicaceae $(61=6.3 \%)$, Chenopodiaceae $(54$ species $=5.6 \%)$ and Caryophyllacea $(39$ species $=4.0 \%)$.
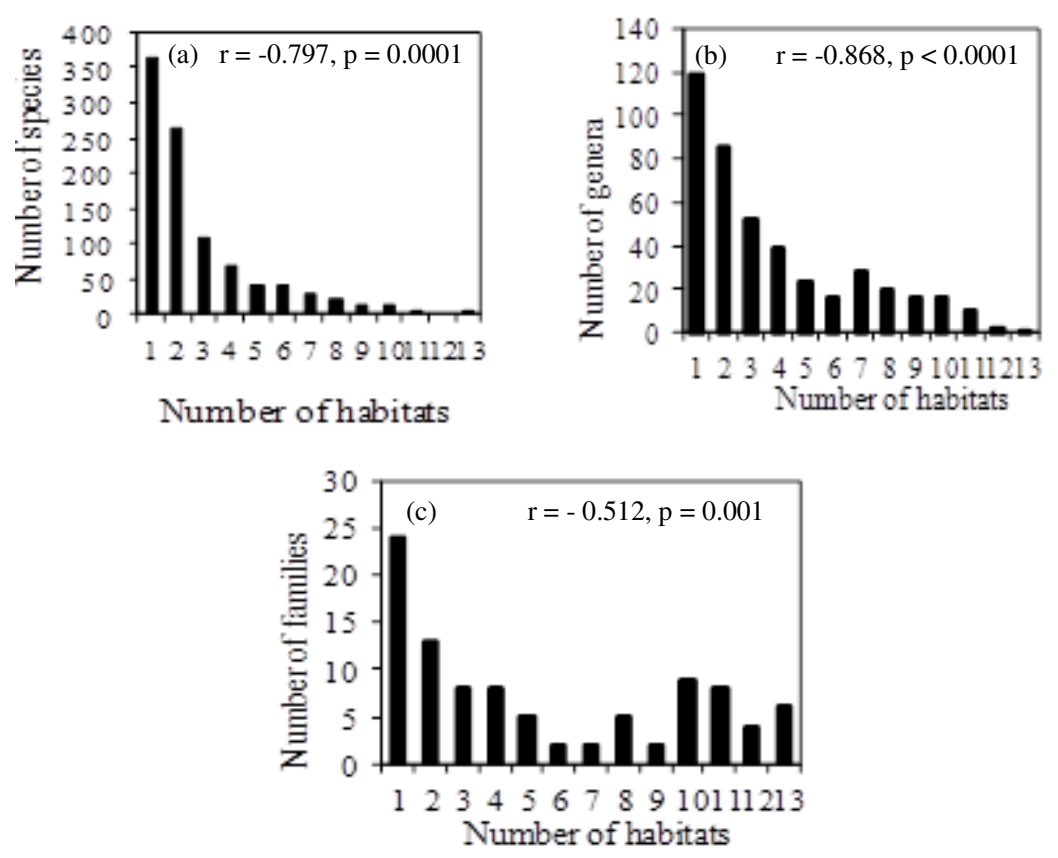

Fig. 2. Number of species (a), genera (b) and families (c) recorded in the Western Mediterranean Desert in relation to the number of habitats in which they occur. 
Taxonomic diversity of the 13 habitats indicated that Sallum plateau had the minimum number of families, genera, species and subspecies (Table. 1). On the other hand, Lake Mariut had the maximum number of families, while the wadis had the maximum of genera, species and subspecies. The ratio of the number of genera to the number of families $(\mathrm{G} / \mathrm{F})$ had the maximum in the wadis and the minimum in non-saline depressions. The ratio of species to genera $(\mathrm{Sp} / \mathrm{G})$ had the minimum value in sand flats and the maximum in non-saline depressions. Finally, the sub-species to species ratio (Subsp/Sp) had the maximum value in sand formations and the minimum in Sallum plateau and road sides.

Table (1). Taxonomic diversity of the 13 major habitats identified in the Western Mediterranean region. The maximum and minimum values are underlined.

\begin{tabular}{|l|c|c|c|c|c|c|c|}
\hline \multicolumn{1}{|c|}{ Habitat } & $\begin{array}{c}\text { Family } \\
(\mathrm{F})\end{array}$ & $\begin{array}{c}\text { Genus } \\
(\mathrm{G})\end{array}$ & $\begin{array}{c}\text { Species } \\
(\mathrm{Sp})\end{array}$ & $\begin{array}{c}\text { Sub- } \\
\text { species }\end{array}$ & $\mathrm{G} / \mathrm{F}$ & $\mathrm{Sp} / \mathrm{G}$ & Sub/Sp \\
\hline Coastal dunes & 44 & 151 & 233 & 38 & 3.4 & 1.5 & 0.16 \\
Sand flats & 30 & 58 & 63 & 11 & 1.9 & $\underline{1.1}$ & $\underline{0.17}$ \\
Sallum plateau & $\underline{20}$ & $\underline{41}$ & $\underline{47}$ & $\underline{6}$ & 2.1 & 1.2 & $\underline{0.13}$ \\
Salt marshes & 21 & 45 & 67 & 10 & 2.1 & 1.5 & 0.15 \\
Saline depressions & 49 & 124 & 198 & 31 & 2.5 & 1.6 & 0.16 \\
Non-saline & 51 & 91 & 322 & 50 & $\underline{1.8}$ & $\underline{3.5}$ & 0.16 \\
Inland ridges & 51 & 184 & 301 & 43 & 3.6 & 1.6 & 0.14 \\
Inland plateau & 44 & 134 & 213 & 31 & 3.0 & 1.6 & 0.15 \\
Wadis & 54 & $\underline{212}$ & $\underline{390}$ & $\underline{59}$ & $\underline{3.9}$ & 1.8 & 0.15 \\
Cultivated lands & 39 & 131 & 205 & 32 & 3.4 & 1.6 & 0.16 \\
Road sides & 45 & 141 & 213 & 28 & 3.1 & 1.5 & $\underline{0.13}$ \\
Summer resorts & 32 & 93 & 130 & 19 & 2.9 & 1.4 & 0.15 \\
Lake Mariut & $\underline{53}$ & 146 & 208 & 29 & 2.8 & 1.4 & 0.14 \\
\hline Total & 97 & 430 & 968 & 138 & 4.4 & 2.3 & 0.14 \\
\hline
\end{tabular}

Life forms of the recorded species (Table 2) indicated that the therophytes $(569$ species $=58.8 \%$ of the total species $)$ were the most represented life form. Of the 140 cryptophytic species ( $14.5 \%$ of the total species), 117 were geo-helophytes (12.1\%), and 23 were hydrophytes $(2.4 \%)$. On the other hand, phanerophytes $(35$ species $=3.6 \%)$ and parasites $(18$ species $=1.9 \%)$ were the less represented life forms. Regarding the flora of the different habitats, phanerophytes and geo-helophytes had the highest relative value in the sandflats. Chamaephytes and hemicryptophytes had the highest relative value in Sallum plateau, parasites in the salt marshes, and therophytes in the summer resorts, while hydrophytes were solely represented in Lake Mariut. 
Table (2). Life form spectra of the recorded species in the 13 habitats identified in the Western Mediterranean region. Ph: phanerophytes, Ch: chamaephytes, $\mathrm{Hm}$ : hemicryptophytes, Ge-He: geo-helophytes, Hy: hydrophytes, Pa: parasites, Th: therophytes.

\begin{tabular}{|c|c|c|c|c|c|c|c|c|}
\hline \multirow{2}{*}{ Habitat } & \multicolumn{7}{|c|}{ Life form } & \multirow{2}{*}{$\begin{array}{c}\text { Total } \\
\text { species }\end{array}$} \\
\hline & $\mathrm{Ph}$ & $\mathrm{Ch}$ & $\mathrm{Hm}$ & $\mathrm{Ge}-\mathrm{He}$ & Hy & $\mathrm{Pa}$ & $\mathrm{Th}$ & \\
\hline Coastal dunes & 13 & 44 & 32 & 22 & - & 2 & 120 & 233 \\
\hline Sand flats & 9 & 16 & 9 & 14 & - & - & 15 & 63 \\
\hline Sallum plateau & 2 & 17 & 11 & 8 & - & - & 9 & 47 \\
\hline Salt marshes & 3 & 14 & 9 & 13 & - & 3 & 25 & 67 \\
\hline Saline depression & 8 & 36 & 25 & 32 & - & 6 & 90 & 198 \\
\hline $\begin{array}{l}\text { Non-saline } \\
\text { depression }\end{array}$ & 9 & 44 & 41 & 48 & - & 2 & 178 & 322 \\
\hline Inland ridges & 11 & 55 & 41 & 36 & - & - & 158 & 301 \\
\hline Inland plateau & 11 & 36 & 22 & 33 & - & 1 & 110 & 213 \\
\hline Wadis & 15 & 58 & 49 & 22 & - & 5 & 241 & 390 \\
\hline Cultivated lands & 4 & 20 & 15 & 21 & - & 6 & 139 & 205 \\
\hline Road sides & 17 & 38 & 25 & 12 & - & 1 & 120 & 213 \\
\hline Summer resorts & 2 & 11 & 7 & 16 & - & 1 & 93 & 130 \\
\hline Lake Mariut & 8 & 18 & 14 & 29 & 21 & 1 & 117 & 208 \\
\hline Total species & 35 & 109 & 97 & 117 & 23 & 18 & 569 & 968 \\
\hline
\end{tabular}

The application of TWINSPAN classification on the floristic composition of the thirteen major habitats identified led to distinguish 6 habitat groups: GI (coastal dunes, sand flats and lake Mariut), which dominated by Ammophila arenaria, Crucianella maritima, Launaea fragilis subsp. fragilis, Echinops spinosus, Deverra tortuosa, Thymelaea hirsuta, Asphodelus aestivus, Phragmites australis subsp. australis and Typha domingensis; GII (saline depressions and salt marshes) dominated by Arthrocnemum macrostachyum, Asphodelus aestivus, Atriplex halimus, Limoniastrum monopetalum, Salsola tetrandra, Sarcocornia fruticusoa and Suaeda monoica; GIII (cultivated lands, Sallum plateau and road sides) dominated by Anabasis articulata, Anagallis arvensis, Deverra tortuosa, Eryngium campestre, Glebionis coronaria, Polygonum equisetiforme and Thymelaea hirsuta; GIV (non-saline depressions and wadis) dominated by Asphodelus aestivus, Capparis spinosa var. inermis, Plantago albicans and Thymelaea hirsuta; GV (inland plateau and inland ridges) dominated by Asphodelus aestivus, Beta vulgaris subsp. maritima, Echinops spinosus, Globularia arabica, Herniaria hirsuta, Malva parviflora and Thymus capitatus and GVI (summer resorts) dominated by Cynodon dactylon and 
Polypogon monspliensis. Most of these groups were well segregated along DECORANA ordination plane (Appendix, Fig. 3).

Many of the recorded species belong to the Mediterranean region (612 species $=63.2 \%$ of the total species), followed by Irano-Turanian (362 species $=37.4 \%)$, Saharo-Arabian $(327$ species $=33.8 \%)$, Euro-Siberian $(167$ species $=17.3 \%)$ and Sudano-Zambezian $(69$ species $=7.1 \%)($ Fig. 4). The general trend of these regions were Pluri-regional $>$ bi-regional $>$ mono-regional species (Table 3). Mediterranean elements had the maximum relative value in the salt marshes, Saharo-Arabian elements in inland plateau, while Sudano-Zambezian elements in Sallum plateau.

A - Habitat classification (TWINSPAN)

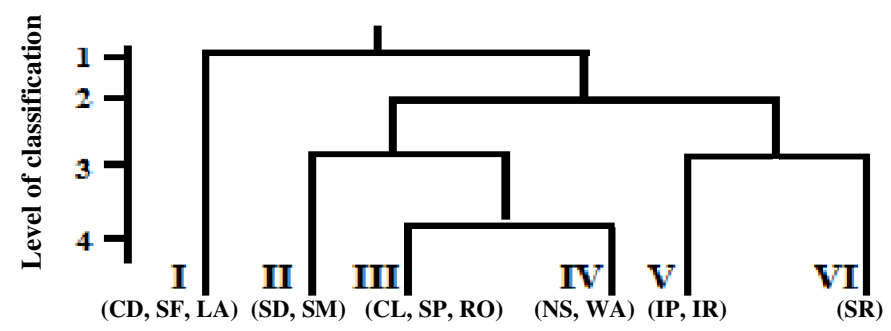

Habitat

B - Habitat ordination (DCA)

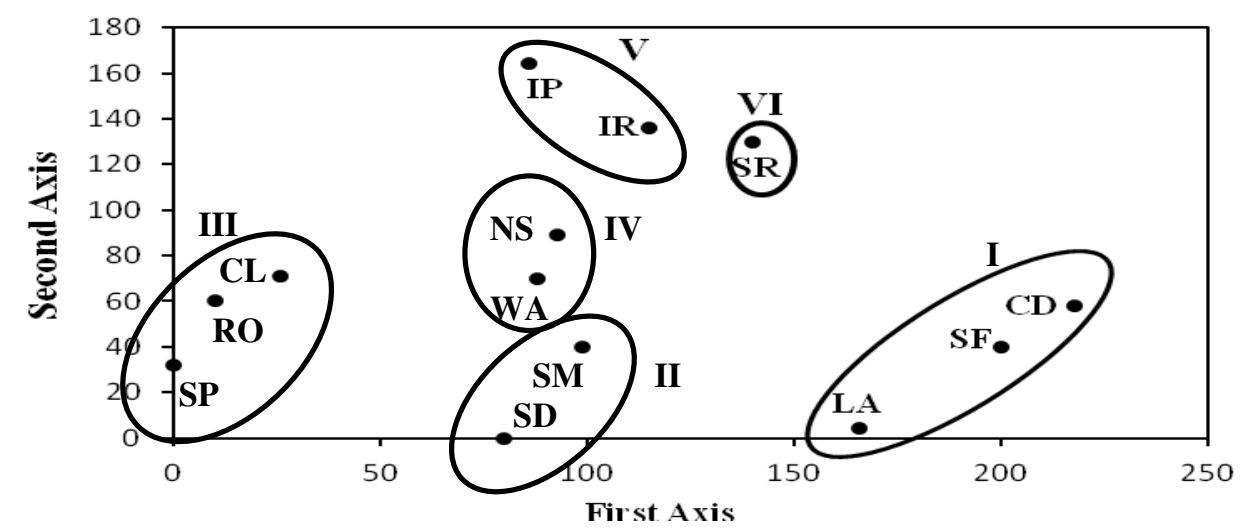

Fig. 3. Classification (A) and ordination (B) of the 13 habitats in the Western Mediterranean region. CD: coastal dunes, SF: sand flats, SP: Sallum plateau, SM: Salt marshes, SD: Saline depressions, NS: Non-saline depressions, IR: Inland ridges, IP: Inland plateaus,WA; Wadis, CL: Cultivated lands, RO: Road sides, SR: Summer resorts and LA: Lake Mariut. 


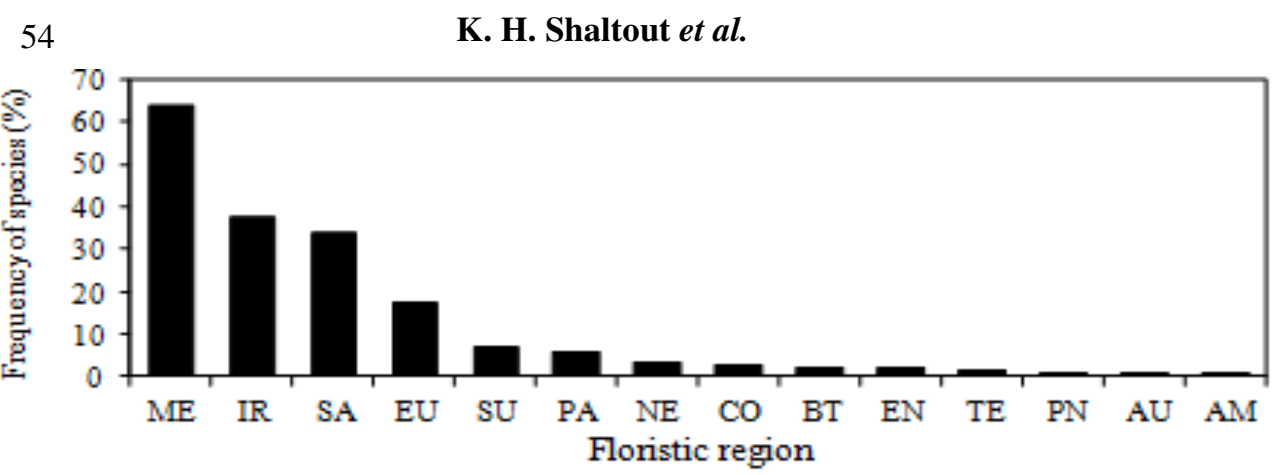

Fig. 4. Frequency of the recorded species in the Western Mediterranean region in relation to their floristic regions. ME: Mediterranean, IR: Irano-Turanian, SA: Saharo-Arabian, EU: Euro-Siberian, SU: Sudano-Zambezian, PA: Palaeotropical, NE: Neotropical, CO: Cosmopolitan, BT: Borealo-Tropical, EN: Endemic species, TE: Temperate, PN: Pantropical, Au: Australian, and AM: America.

Ninteen endemics and 21 near endemics were recorded in the study area (Table 4). Nine endemic species were restricted to one habitat, 8 species to 2 habitats. Regarding the variation in relation to the phytogeographical regions and abundance categories, 9 of very rare endemics were unique to the study area while 3 rare endemics were recorded in two phytogeographical regions On the other hand, 6 endemics were common and had a wide geographical distribution.

Twenty one near-endemics were restricted to Egypt, Palestine and Libya. Regarding the variation in relation to the habitat types, 4 species were restricted to one, 6 in two habitats 2 in three habitats ( 2 in four habitats 3 in five habitats one species in six habitats (Centaurea alexandrina), 2 in seven habitatsand one in nine habitats (Centaurea glomerata).

Regarding the variation in relation to phytogeographical regions and abundance categories 3 of them were unique and recorded as very rare species (Crepis libyca, Euphorbia parvula and Valantia lanata), 5 species were recorded in two phytogeographical regions and recorded as rare species. On the other hand 2 species had a wide phytogeohraphical distribution and recorded as common species, while only 2 species were recorded as very common species recorded only in two phytogeographical regions (Table 4). 
Table (3). Floristic regions in relation to the 13 habitats identified in the Western Mediterranean region. CD: coastal dunes, SF: sand flats, SP: sallum plateau, SM: salt marshes, SD: saline depression, NS: non-saline depression, IR: inland ridges, IP: inland plateau, WA: wadis, CL: cultivated lands, RO: road sides, SR: Summer resorts and LA:lake Mariut. ME: Mediterranean, IR-TR: Irano-Turanian, SA-AR: Saharo-Arabia, ER-SR: Euro-Siberian, SU-ZA: Sudano-Zambezian, COSM: Cosmopolitan, PAL: Palaeotropical, NEO: Neotropical and Aust.: Australian.

\begin{tabular}{|c|c|c|c|c|c|c|c|c|c|c|c|c|c|}
\hline \multirow{2}{*}{ Floristic region } & \multicolumn{13}{|c|}{ Habitat } \\
\hline & $\mathrm{CD}$ & $\mathrm{SF}$ & SP & SM & SD & NS & IR & IP & WA & $\mathrm{CL}$ & RO & SR & LA \\
\hline \multicolumn{14}{|l|}{ Mono-regional } \\
\hline Endemics & 3 & - & - & - & 2 & 9 & 7 & 3 & 5 & 4 & 1 & - & 5 \\
\hline ME & 36 & 10 & 4 & 13 & 31 & 51 & 49 & 33 & 56 & 31 & 28 & 8 & 35 \\
\hline SA-AR & 54 & 8 & 21 & 7 & 44 & 77 & 61 & 55 & 73 & 25 & 39 & 5 & 16 \\
\hline SU-ZA & 2 & 1 & 1 & - & 1 & - & 1 & - & 2 & 1 & 3 & 1 & 4 \\
\hline IR-TR & 1 & - & - & - & 2 & 2 & 1 & 1 & 2 & 1 & - & - & - \\
\hline \multicolumn{14}{|l|}{ Bi-regional } \\
\hline $\mathrm{ME}+\mathrm{SA}-\mathrm{AR}$ & 35 & 12 & 9 & 7 & 28 & 37 & 35 & 22 & 36 & 19 & 28 & - & 16 \\
\hline $\mathrm{ME}+\mathrm{IR}-\mathrm{TR}$ & 17 & 6 & 3 & 4 & 20 & 38 & 37 & 20 & 56 & 33 & 18 & 15 & 25 \\
\hline $\mathrm{ME}+\mathrm{ER}-\mathrm{SR}$ & 8 & 1 & - & 4 & 5 & 4 & 4 & 1 & 12 & 6 & 7 & 7 & 18 \\
\hline $\mathrm{ME}+\mathrm{SU}-\mathrm{ZA}$ & 1 & 1 & - & - & - & - & 1 & - & 1 & - & 1 & - & - \\
\hline SA-AR + SU-ZA & 8 & 5 & 1 & 1 & 9 & 10 & 11 & 15 & 12 & 3 & 10 & 2 & 7 \\
\hline IR-TR + SU-ZA & - & - & - & - & - & - & - & - & - & - & 1 & 1 & 1 \\
\hline SA-AR + ER-SR & - & - & - & - & - & 1 & 1 & - & - & - & - & - & - \\
\hline IR-TR + SA-AR & 19 & 3 & 1 & 2 & 14 & 23 & 23 & 18 & 25 & 4 & 10 & 2 & 10 \\
\hline \multicolumn{14}{|l|}{ Pluri-regional } \\
\hline COSM & 1 & - & 1 & 3 & 3 & 4 & 3 & 2 & 2 & 4 & 4 & 13 & 24 \\
\hline PAN & 1 & - & - & - & 1 & 1 & 1 & 3 & 1 & 3 & - & 2 & 3 \\
\hline PAL & - & 2 & - & 1 & 2 & 3 & 3 & 3 & 3 & 4 & 2 & 6 & 11 \\
\hline NEO & 1 & - & - & - & - & 1 & - & - & 2 & 1 & 2 & 4 & 7 \\
\hline Temperate & - & - & - & - & - & 1 & 1 & - & 1 & 1 & 2 & 2 & 1 \\
\hline Aust. & 1 & - & - & - & - & - & - & - & 1 & 1 & - & - & 1 \\
\hline America & - & - & - & - & - & - & - & - & - & 1 & - & - & - \\
\hline Borealo-Tropical & 2 & 2 & 1 & 4 & 2 & 3 & 4 & 2 & 5 & 3 & 3 & 7 & 14 \\
\hline Temperate+NEO & - & - & - & - & - & - & - & - & 1 & - & 1 & 1 & - \\
\hline Temperate+ME & - & - & - & - & 1 & - & - & - & - & 1 & - & - & 2 \\
\hline $\mathrm{ME}+\mathrm{PAL}$ & - & - & - & - & - & - & - & - & 1 & - & - & 2 & 1 \\
\hline $\mathrm{ME}+\mathrm{NEO}$ & - & - & - & - & - & - & - & - & 2 & - & 1 & 1 & 1 \\
\hline $\mathrm{ME}+$ Aust & - & - & - & - & - & - & - & - & - & - & - & - & 1 \\
\hline $\mathrm{PAL}+\mathrm{NEO}$ & 1 & 1 & 1 & - & 1 & 1 & 1 & 1 & 2 & 3 & 2 & 4 & 5 \\
\hline $\mathrm{ME}+\mathrm{IR}-\mathrm{TR}+\mathrm{ER}-\mathrm{SR}$ & 18 & 4 & 1 & 7 & 9 & 25 & 23 & 9 & 47 & 31 & 25 & 26 & 63 \\
\hline ME+IR-TR+ SU-ZA & - & - & - & - & 1 & - & 1 & 1 & 1 & - & - & - & - \\
\hline ME+ER-SR +SA-AR & 3 & 1 & - & 1 & 2 & 2 & 2 & 2 & 2 & 1 & 2 & - & 3 \\
\hline $\mathrm{ME}+\mathrm{SU}-\mathrm{ZA}+\mathrm{SA}-\mathrm{AR}$ & 5 & 2 & 1 & - & 1 & 6 & 6 & 5 & 2 & 5 & 2 & 2 & - \\
\hline $\mathrm{ME}+\mathrm{PAL}+\mathrm{NEO}$ & - & - & - & - & - & - & - & - & 1 & 1 & - & 1 & 2 \\
\hline $\mathrm{ME}+\mathrm{IR}-\mathrm{TR}+\mathrm{PAL}$ & 1 & - & - & 2 & 1 & - & - & - & - & 1 & - & 1 & 2 \\
\hline
\end{tabular}


K. H. Shaltout et al.

Table 3. cont.

\begin{tabular}{|c|c|c|c|c|c|c|c|c|c|c|c|c|c|}
\hline \multirow{2}{*}{ Floristic region } & \multicolumn{13}{|c|}{ Habitat } \\
\hline & $\mathrm{CD}$ & SF & SP & $\mathrm{SM}$ & $\mathrm{SD}$ & NS & IR & IP & WA & CL & RO & SR & LA \\
\hline $\mathrm{ME}+\mathrm{IR}-\mathrm{TR}+\mathrm{SA}-\mathrm{AR}$ & 9 & 3 & - & 4 & 8 & 13 & 16 & 6 & 18 & 10 & 9 & 7 & 7 \\
\hline ME+IR-TR +Aust. & - & - & - & 1 & 2 & - & - & - & - & - & - & - & 1 \\
\hline IR-TR+SU-ZA+SA-AR & 2 & 1 & 1 & 1 & 2 & 3 & 2 & 2 & 3 & 2 & 2 & - & 1 \\
\hline $\mathrm{ME}+\mathrm{IR}-\mathrm{TR}+\mathrm{PAL}+\mathrm{NEO}$ & - & - & - & 2 & 2 & - & - & 2 & 1 & 3 & - & 2 & 3 \\
\hline $\begin{array}{l}\text { ME+IR-TR+ER-SR+ } \\
\text { Temperate }\end{array}$ & - & - & - & - & - & - & - & - & - & - & 1 & - & - \\
\hline $\mathrm{ME}+\mathrm{IR}-\mathrm{TR}+\mathrm{ER}-\mathrm{SR}+\mathrm{PAL}$ & - & - & - & - & - & - & - & - & 3 & 1 & - & 1 & - \\
\hline $\mathrm{ME}+\mathrm{IR}-\mathrm{TR}+\mathrm{ER}-\mathrm{SR}+\mathrm{SA}-\mathrm{AR}$ & - & 1 & 1 & 1 & 2 & 3 & 2 & 2 & 4 & - & 6 & 2 & 3 \\
\hline IR-TR+ER-SR+SU-ZA & - & - & - & - & - & - & - & - & - & - & - & 1 & - \\
\hline $\mathrm{ME}+\mathrm{IR}-\mathrm{TR}+\mathrm{SA}-\mathrm{AR}+\mathrm{PAL}$ & 1 & - & - & - & - & 1 & - & - & - & - & - & - & - \\
\hline $\begin{array}{l}\mathrm{ME}+\mathrm{IR}-\mathrm{TR}+\mathrm{SA}-\mathrm{AR} \\
+\mathrm{PAL}+\mathrm{NEO}\end{array}$ & 1 & - & - & - & - & - & - & - & 1 & 1 & - & 5 & 1 \\
\hline $\begin{array}{l}\mathrm{ME}+\mathrm{IR}-\mathrm{TR}+\mathrm{PAL}+\mathrm{NEO} \\
+ \text { Aust. }\end{array}$ & - & - & - & - & - & - & - & - & - & - & - & 1 & - \\
\hline $\mathrm{ME}+\mathrm{IR}-\mathrm{TR}+\mathrm{SA}-\mathrm{AR}+\mathrm{SU}-\mathrm{ZA}$ & 1 & - & - & 1 & 1 & 2 & 3 & 2 & 2 & - & 1 & - & - \\
\hline SA-AR+SU-ZA+PAL & - & - & - & - & - & - & - & 1 & - & - & - & - & - \\
\hline $\begin{array}{l}\mathrm{ME}+\mathrm{IR}-\mathrm{TR}+\mathrm{ER}-\mathrm{SR}+\mathrm{PAL}+ \\
\text { Temperate }\end{array}$ & - & - & - & - & - & - & - & - & 1 & - & 1 & - & - \\
\hline $\mathrm{ME}+\mathrm{IR}-\mathrm{TR}+\mathrm{ER}-\mathrm{SR}+\mathrm{SU}-\mathrm{ZA}$ & 1 & - & - & 1 & 1 & 1 & 2 & 2 & 3 & - & 1 & 1 & 3 \\
\hline $\mathrm{ME}+\mathrm{IR}-\mathrm{TR}+\mathrm{NEO}$ & - & - & - & - & - & - & - & - & - & - & - & - & 1 \\
\hline Total & 233 & 64 & 47 & 67 & 198 & 322 & 301 & 213 & 390 & 205 & 213 & 130 & 299 \\
\hline
\end{tabular}


Table (4). Endemic and near endemic species recorded in the Western Mediterranean region. The code of habitats are: IR: inlandridges, NS: non-saline depressions: $\mathrm{Cl}$ : cultivated lands, WA: wadis, LA: lake Mariut, CD: coastal dunes, SF: sand formations, SM: salt marshes, IP: inland plateaus, SD: saline depressions and RO: road sides. The code of phytogeographical regions are: Mma: Western Mediterranean coastal region, Mp: Eastern Mediterranean coastal region, Di: Isthmic desert, Nd: Nile Delta, Nv: Nile Valley, Nf: Nile Faiyum, Da: Eastern desert, Dl: Libyan desert and O: Oases of western desert, R: Red Sea coastal region and S: Sinai proper. The dominance magnitudes as: cc: very common, c: common, r: rare and rr: very rare.

\begin{tabular}{|c|c|c|c|}
\hline Species & Family & Habitat & Phytogeographical regions \\
\hline \multicolumn{4}{|l|}{ a - Endemics } \\
\hline Allium blomfieldianum & Alliaceae & IR & Mma (rr) \\
\hline Allium mareoticum & Alliaceae & IR, NS & $\mathrm{Mma} \quad(\mathrm{rr})$ \\
\hline Anthemis microsperma & Compositae & CD, NS, IR, CL, WA & Mma, Mp (c) \\
\hline Atractylis carduus var. marmarica & Compositae & $\begin{array}{l}\mathrm{CD}, \mathrm{SD}, \mathrm{NS}, \mathrm{IR}, \mathrm{IP}, \mathrm{CL} \\
\mathrm{WA}, \mathrm{RO}\end{array}$ & Mma, Di (r) \\
\hline Bellevalia romana & Hyacinthaceae & $\mathrm{CL}$ & Mma (rr) \\
\hline Bellevalia salah-eidii & Hyacinthaceae & IR, NS & Mma (rr) \\
\hline Bromus aegyptiacus & Gramineae & LA & Nd,Nv,Nf,Mma (c) \\
\hline Ebenus armitagei & Leguminosae & IR, NS & $\mathrm{Mma} \quad(\mathrm{rr})$ \\
\hline $\begin{array}{l}\text { Eragrostis aegyptiaca subsp. } \\
\text { aegyptiaca }\end{array}$ & Gramineae & CL, NS & Nd,Nv,Mma,Mp,Di,Da,Dl (c) \\
\hline Euphorbia punctata & Euphorbiaceae & WA & Mma (rr) \\
\hline Fumaria microstachys & Fumariaceae & LA & Mma (rr) \\
\hline $\begin{array}{l}\text { Limonium sinuatum } \quad \text { subsp. } \\
\text { ramanum }\end{array}$ & Plumbaginaceae & SD & Mma (rr) \\
\hline Muscari albiflorum & Hyacinthaceae & NS, IP & Mma (rr) \\
\hline Pancratium arabicum & Amaryllidaceae & $\mathrm{CD}$ & Mma, Mp (r) \\
\hline Silene biappendiculata & Caryophyllaceae & $\mathrm{IR}, \mathrm{NS}$ & Nd, Mma, Mp, Di (c) \\
\hline Sinapis allionii & Cruciferae & LA & $\mathrm{Nd}, \mathrm{Nv}, \mathrm{O}, \mathrm{Mma}, \mathrm{Mp}$ (c) \\
\hline Sonchus macrocarpus & Compositae & CL, WA & $\mathrm{Nd}, \mathrm{Mma} \quad(\mathrm{r})$ \\
\hline Thesium humile var. maritima & Santalaceae & WA & Mma (c) \\
\hline Trigonella occulta & Leguminosae & NS, IP & Nv, Nf, Mma, Di (r) \\
\hline \multicolumn{4}{|l|}{ B - Near endemics } \\
\hline Aegilops longissima & Gramineae & $\mathrm{RO}, \mathrm{CL}$ & Mma, Mp (r) \\
\hline Allium desertorum & Alliaceae & $\mathrm{SD}, \mathrm{NS}, \mathrm{IR}, \mathrm{IP}$ & Mma, Di, Dl (r) \\
\hline Anthemis indurata & Compositae & NS,IR & Mma, Mp, Di (r) \\
\hline Bellevalia eigii & Hyacinthaceae & NS,IR & Mma, Mp (rr) \\
\hline Biarum olivieri & Araceae & NS,IP & Mma, Mp (rr) \\
\hline $\begin{array}{l}\text { Lycium schweinfurthii subsp. } \\
\text { aschersohnii }\end{array}$ & Solanaceae & CD,IR,IP,SD,SM & Nd, Mma, Di (r) \\
\hline Onopordum alexandrinum & Compositae & $\mathrm{SF}, \mathrm{CD}, \mathrm{WA}, \mathrm{RO}$ & Mma, Mp (cc) \\
\hline
\end{tabular}


Table 4. Cont.

\begin{tabular}{|c|c|c|c|}
\hline Species & Family & Habitat & Phytogeographical regions \\
\hline Plantago crypsoides & Plantaginaceae & $\begin{array}{l}\text { CD, NS, SD, IR, IP, } \\
\text { WA, RO }\end{array}$ & Nd, Mma, Da, Di, Dl (c) \\
\hline \multicolumn{4}{|l|}{ Egypt and Libya } \\
\hline Allium barthianum & Alliaceae & WA,SP & $\operatorname{Mma}(\mathrm{r})$ \\
\hline Bellevalia sessiliflora & Hyacinthaceae & $\mathrm{SP}, \mathrm{IR}, \mathrm{NS}$ & Mma, Mp (c) \\
\hline Bupleurum nanum & Umbelliferae & $\mathrm{SD}, \mathrm{IR}, \mathrm{RO}$ & Mma, Mp (r) \\
\hline Carduncellus mareoticus & Compositae & SP, SD, NS, IP, LA & Mma, Di (r) \\
\hline Carthamus glaucus & Compositae & SP, SD, IR, RF, WA & Mma, Mp (r) \\
\hline Centaurea alexandrina & Compositae & $\begin{array}{l}\text { CD, WA, NS, IR, IP, } \\
\text { RO }\end{array}$ & Mma, Mp (cc) \\
\hline Centaurea glomerata & Compositae & $\begin{array}{l}\text { SF, CD, SD, NS, IR, } \\
\text { IP. RF. WA. RO }\end{array}$ & Nd, O, Mma, Mp, Di, Dl (c) \\
\hline Crepis libyca & Compositae & NS & Mma (rr) \\
\hline Euphorbia parvula & Euphorbiaceae & WA & Mma (rr) \\
\hline Ferula marmarica & Umbelliferae & IR & Mma, Di (rr) \\
\hline Helianthemum sphaerocalyx & Cistaceae & $\mathrm{CD}$ & Mma, Di (r) \\
\hline Valantia lanata & Rubiaceae & WA, IR & Mma (rr) \\
\hline Verbascum letourneuxii & Scrophulariaceae & $\begin{array}{l}\mathrm{SP}, \mathrm{CD}, \mathrm{SD}, \mathrm{NS}, \mathrm{IP}, \\
\mathrm{WA}, \mathrm{RO}\end{array}$ & Mma, Di (r) \\
\hline
\end{tabular}

The majority of species ( 599 species $=61.9 \%$ of the total species $)$ had small geographical distribution, narrow habitat specificity and were nonabundant (SNN) (Table 5). On the other hand, 23 species only (2.4\% of the total species) had large geographical distribution, wide habitat specificity and non-abundant (LWN). Regarding the variation in relation to habitats, The most common species (i.e. LWA and LWN) had the maximum relative value in sand formation and Sallum plateau, respectively. LNA species were concentrated in the summer resorts, LNN in the salt marshes, SWN in sallum plateau, and SNN in the cultivated lands.

Forty one species were recorded as introduced species to the Egyptian flora (Table 6), most of them were therophytes $(57.1 \%$ of the total naturalized species), and followed by phanerophytes (16.7\%). On the other hand, hemicryptophytes and parasites were less represented (each of them represented by $2.4 \%$ ). Some of these species could consider as invasive species such as Azolla filiculoides, Bassia indica, Eichhornia crassipes, Ipomoea carnea, Paspalum distichum and Vossia cuspidata. 
Table 5. Spectra of the rarity forms of the recorded species in relation to their habitats in the Western Mediterranean region. A abundant, N: non-abundant.

\begin{tabular}{|c|c|c|c|c|c|c|c|}
\hline \multirow{4}{*}{ Habitat } & \multirow{4}{*}{$\begin{array}{l}\text { Total } \\
\text { species }\end{array}$} & \multicolumn{6}{|c|}{ Rarity form } \\
\hline & & \multicolumn{4}{|c|}{ Large geographical gradient } & \multicolumn{2}{|c|}{ Small geographical gradient } \\
\hline & & \multicolumn{2}{|c|}{ Wide habitat } & \multicolumn{2}{|c|}{ Narrow habitat } & Wide habitat & Narrow habitat \\
\hline & & A & $\mathrm{N}$ & A & $\mathrm{N}$ & $\mathrm{N}$ & $\mathrm{N}$ \\
\hline Coastal dunes & 233 & 37 & 19 & 19 & 21 & 43 & 94 \\
\hline Sand formation & 63 & 21 & 10 & 2 & 1 & 14 & 15 \\
\hline Sallum plateau & 47 & 10 & 8 & 3 & 5 & 11 & 10 \\
\hline Salt marshes & 67 & 5 & 4 & 10 & 12 & 7 & 29 \\
\hline Saline depressions & 198 & 26 & 13 & 21 & 19 & 35 & 84 \\
\hline Non-saline depressions & 322 & 42 & 21 & 26 & 26 & 47 & 160 \\
\hline Inland ridges & 301 & 43 & 22 & 18 & 22 & 46 & 150 \\
\hline In land plateaus & 213 & 28 & 16 & 18 & 18 & 38 & 95 \\
\hline Wadis & 390 & 47 & 21 & 41 & 38 & 42 & 201 \\
\hline Cultivated lands & 205 & 33 & 13 & 17 & 13 & 22 & 107 \\
\hline Road sides & 213 & 38 & 17 & 24 & 12 & 34 & 88 \\
\hline Summer resorts & 130 & 25 & 5 & 40 & 15 & 3 & 42 \\
\hline Lake Mariut & 208 & 25 & 5 & 45 & 28 & 9 & 96 \\
\hline Total & 968 & 50 & 24 & 134 & 112 & 49 & 599 \\
\hline
\end{tabular}

Table 6. Introduced species in the Western Mediterranean Desert.

\begin{tabular}{|c|c|c|c|}
\hline Name & Family & Name & Family \\
\hline Phanerophytes & & Bassia indica & Chenopodiaceae \\
\hline Atriplex nummularia & Chenopodiaceae & Bidens pilosa & Compositae \\
\hline Nicotiana glauca & Solanaceae & Bromus catharticus & Gramineae \\
\hline Rubus sanctus & Rosaceae & Cenchrus echinatus & Gramineae \\
\hline Salix tetrasperma & Salicaceae & Chenopodium glaucum & Chenopodiaceae \\
\hline Sesbania sesban & Leguminosae & Chloris gayana & Gramineae \\
\hline Ziziphus spina-christi & Rhamnaceae & Datura innoxia & Solanaceae \\
\hline Chamaephytes & & Datura metel & Solanaceae \\
\hline Asclepias fruticosa & Asclepiadaceae & Datura stramonium & Solanaceae \\
\hline Chenopodium ambrosioides & Chenopodiaceae & Euphorbia heterophylla & Euphorbiaceae \\
\hline Ipomea carnea & Convolvulaceae & Euphorbia hirta & Euphorbiaceae \\
\hline Ononis vaginalis & Leguminosae & Euphorbia prostrata & Euphorbiaceae \\
\hline Symphyotrichum squamatum & Compositae & Gynandropsis gynandra & Cleomaceae \\
\hline Hemicryptophytes & & Lathyrus sativus & Cruciferae \\
\hline Euphorbia mauritanica & Euphorbiaceae & Lepidium sativum & Cruciferae \\
\hline Geo-Helophytes & & Matricaria recutita & Compositae \\
\hline Oxalis pes-caprae & Oxalidaceae & Mesembryanthemum crystallinum & Aizoaceae \\
\hline Paspalum distichum & Gramineae & Phalaris canariensis & Gramineae \\
\hline Vossia cuspidata & Gramineae & Securigera securidacea & Leguminosae \\
\hline \multicolumn{4}{|l|}{ Therophytes } \\
\hline $\begin{array}{l}\text { Amaranthus hybridus subsp. } \\
\text { hybridus }\end{array}$ & Amaranthaceae & Hydrophytes & \\
\hline Argemone mexicana & Papaveraceae & Azolla filiculoides & Azollaceae \\
\hline Atriplex holocarpa & Chenopodiaceae & Eichhornia crassipes & Pontederiaceae \\
\hline Atriplex lindleyi subsp. inflata & Chenopodiaceae & Parasites & \\
\hline Atriplex semibaccata & Chenopodiaceae & Cuscuta campestris & Cuscutaceae \\
\hline
\end{tabular}




\section{Discussion}

The total number of vascular plant species recorded in the present study is 968, related to 429 genera and 97 families, Asteraceae (126 species), Poaceae (119 species) and Fabaceae (114 species) and were represented together by 359 species or about $37.1 \%$ of the total number of the recorded species. The highest represented families in the Egyptian flora (Boulos 2009) were Fabaceae (Leguminosae), Poaceae (Gramineae) and Asteraceae (Compositae), which together account for $34 \%$ of the flora. Cruciferae (61 species), Chenopodiaceae (54 species), and Caryophyllaceae (39 species) comprise $15.9 \%$ of the total number of species. This indicates that the previous six families were loading taxa and constitute the main bulk of the flora of the Western Mediterranean region (Khedr et al. 2002). This finding agrees with that of Quézel (1978) concerning the floristic structure of North Africa. The flora of Egypt as indicated in Boulos (2009) represented by 2145 species, related to 755 genera and 129 families (including some cultivated taxa); this means that the flora of the Western Mediterranean region contributes $45.1 \%$ of the total species, $56.8 \%$ of the total genera and $75.2 \%$ of the total families. Comparing with the other phytogeographical regions of Egypt, Western Mediterranean region comes after Sinai region (1310 species), while it is richer than the Deserts (869 species), Oases (476 species), Gebel Elba (451 species) and Red Sea (308 species) regions (as calculated by the author from the database of Tanta University Herbarium (TANE), taking into account Boulos $(1999,2000,2002,2005)$. The north western coastal region is by far the richest part of Egypt in its floristic composition owing to its relatively high rainfall. were a great numberof individual plants and the vegetation is more or less continuous, not like that in the inland desert areas, where the plant communities were separated by large stretches of barren ground (Zahran and Willis 2009).

Zohary (1973) stated that the striking feature in the Egyptian flora is the large number of genera in proportion to that of species, $(2.9$ according to Boulos 2009). This is a very low figure compared with the average global proportion, which is about 13.6. The present study indicated that the flora of the Western Mediterranean region goes below the average level of the Egyptian flora, where the number of species per genus is 2.3. This means that the flora of the Western Mediterranean region is floristically more diverse than the whole Egyptian flora, as the region that has a certain number of species, each of which belongs to a different genus, is relatively more 
diverse than a region with the same number of species but belong to a few number of genera (Hawksworth, 1995; Shaltout et al., 2010).

The well known 'life form' system is perhaps that of Raunkiaer (1937), which was designed for the northern temperate zone, and based on the location of renewal buds. The life form spectrum is thought to be either hereditary adjustment to environment (El-Demerdash, 1984), or representing the residual effects of some historical, climatic or biotic conditions on the plant population (Waisel, 1972). In the present study, the therophytes were the most frequent life form in the study area, followed by the cryptophytes and chamaephytes. The dominance of therophytes over the other life forms seems to be a response to the hot-dry climate, topographic variation and biotic influence (Heneidy and Bidak, 2001). The short life cycles of field crops (the new land use at present were rainfed farming: Ayyad, 1983), in addition to the adverse climatic conditions, moisture deficiency and substrate instability probably lead to the frequent occurrence of therophytes during the favorable seasons. Ayyad and El-Ghareeb (1982) reported that chamaephytes and therophytes form about $70 \%$ of the vegetation of the Egyptian western Mediterranean salt marshes, while Zahran and Wills (2003) argued that chamaephytes and cryptophytes were the most abundant life forms in halophytic vegetation in Egypt. El-Demerdash (1984) and Shaltout \& ElGhareeb (1992) reported that the predominance of the cryptophytes in the salt marshes is probably attributed to their growth habitats. Most of these plants were rhizomatous (e.g. Juncus rigidus, Phragmites australis and Aeluropus lagopoides), which were resistant to decomposition under constant submergence. In addition, the less compact sandy soil of the salt marshes is easily pentrable by the rhizomes of cryptophytes (El-Demerdash et al. 1987).

Floristic analysis revealed that the Mediterranean element (about 63.2 $\%$ of the total species) is the most represented in the Western Mediterranean region. According to (Kosinová, 1972), the important part of the recent synanthropic flora of Egypt has a Mediterranean origin or distribution. However, the Mediterranean species range corresponds to two climatic belts, viz. the Mediterranean coastal belt and Middle Egypt (Kassas 1955). The Mediterranean vegetation is dominated by evergreen sclerophyllous shrubs that form maquis (over $2 \mathrm{~m}$ in height), garrigue and jaral $(0.6-2 \mathrm{~m})$, phrygana or batha $(<0.6 \mathrm{~m})$ plant communities (Archibold, 1995). In general, the Mediterranean areas were defined according to temperature 
conditions (mean annual range is $10-25^{\circ} \mathrm{C}$ ), but the precipitation regime is the most distinctive $\left(275-900 \mathrm{~mm}^{-1} \mathrm{ye}^{-1}\right.$ with at least $65 \%$ falling during winter) (Dallman, 1998). In the present study, a mixture of different floristic elements such as Cosmopolitan, Pantropical, Palaeotropical, Neotropical, Saharo-Arabian, Sudano-Zambezian, Irano-Turanian and Euro-Siberian elements were represented by variable numbers of species. This can be attributed to human impact, agriculture and capability of certain floristic elements to penetrate the study area from several adjacent phytogeographical regions (Seif El-Nasr and Bidak, 2006).

Sixty endemic species were recorded from Egypt, the Sinai Peninsula has about 39 species (65\% of the Egyptian endemic species) in its southern mountainous region In the Western Mediterranean region, 18 endemic species were recorded in the present study (Boulos, 2009) in addition to Eragrostis aegyptiaca subsp. aegyptiaca which was recorded by Cope and Hosni (1991).

Rabinowitz (1981) indicated the different types of rarity depending on range, habitat specificity and local abundance. In the present study, LWN group contains 24 species $(2.5 \%$ of the total species $)$; this is probably the most ignored category of inconspicuous and unspectacular plants, with large ranges, several habitats, but of consistently low populations. On the other hand, no species belong to SWA group, while 49 species (5.1\% of the total) belong to SWN; this is may be revert to demographic stochasticity, which is a process in small populations analogous to genetic drift, and which in fluctuating population numbers, due to small sample phenomena, may cause local extinction (Rabinowitz, 1981). These deletions of populations may reduce the variety of habitats occupied and in essence, convert perhaps an unstable species into one in the categories SNA and SNN (Rabinowitz, 1981).

Five hundred and ninety nine species in the present study $(61.9 \%$ of the total species) belong to SNN group. Species with both small geographic range and narrow habitat specificity (SNA and $\mathrm{SNN}$ ) were the classic rarities in the sense of restricted endemics, often endangered or threatened. Species which have large ranges but were associated with particular habitats were generally quite predictable in their occurrence (LNA and LNN), these species tend to be precarious as a result of habitat destruction (Rabinowitz, 1981). The percentage of rare species (SWN and SNN) approximates $67 \%$ of the flora of the region, whereas the common species (LWA, LWN, LNA 
and $\mathrm{LNN}$ ) attained $33 \%$, this finding is very important and represent an alarm to consider conservation of natural vegetation of the study area as a must. According to Grime (1974), families with a greater number of rare species were more stress-tolerant. Those with many common species tend to exhibit competitive or competitive-ruderal strategy and often have several alternative strategies for regeneration.

The history of plant introduction has been recorded by the activities of dedicated botanists who collected, presses, mounted and archived plants in herbaria. This work has turned out to be a goldmine for ecologists seeking to understand the patterns of spread of introduced species (Judith and Dawn 2003). In Britan, Crawley et al. (1996) reported that $46 \%$ of its flora (2684 species) as exotics, In European flora (1568 species were considered as aliens), $37 \%$ were exotic species and $63 \%$ naturalized species (Weber, 1997). Introduced species to the Egyptian flora were represented by 137 species which contribute about $6.2 \%$ of its natural flora (Shaltout, 2014). These species were mostly weeds and differ from each other not only in the time and way of their introduction, but also in the degree of establishment in natural and human-made habitats (Simpson, 1932; Drar, 1952, El-Hadidi and Kosinová, 1971; Täckholm, 1974; Täckholm and Boulos, 1974; Hejny and Kosinová, 1977; Boulos 2009). The low number of introduced species in the Egyptian flora does not reflect the actual number, as in a country of an ancient human civilization like Egypt, it is difficult to decide definitely if many of its weed flora have been originated from the native flora or introduced by human activity (Hassib, 1951; El-Fahar and Sheded, 2002). The assessment of the introduced species into Egypt needs further studies. As an example, in addition to the 137 recently-introduced species (Shaltout, 2014), Fahmy (1997) presented a list of 57 species (46 of them occur in the checklist of the Western Mediterranean region) seem to be introduced into the fields as weeds during the Predynastic Period (4500 B.C.).

Out of the 137 introduced species to the Egyptian flora, 41 species were recorded in the present study (30.7\% of the total naturalized species). Exotics may become invasive and outcompete natives (Judith and Dawn, 2003). Paspalum distichum, one of the naturalized species in the present study, was introduced from America and rapidly spread in the Western Mediterranean Desert during early decades of the past century. It became naturalized lately in other parts of the Egyptian cultivated land and well established in artificial and semi natural habitats (Hejny and Kosinová, 
1977). Ipomoea carnea, one of the invasive exotics, was introduced into Egypt as an ornamental plant. It is native to South America and was reported as naturalized species along the water courses in the Western Mediterranean region (El-Fahar and Sheded, 2002; Shaltout and Galal, 2006). Bassia indica (Syn. Kochia indica) is another invasive species in the study area. It is native to south and east India and was introduced into Egypt in 1945 as a promising fodder plant to fill a gap in the ranges of the north western coastal strip of Egypt. After that, it began to invade the Nile Delta and other related regions of the Nile valley (Drar, 1952; El-Fahar, 1989; Al-Sodany, 1992, 1998; Shaltout and El-Beheiry, 2000; Shaltout et al. 2010).

Azolla filiculoides is a fern that had escaped from an experimental field, which was carried out to be used for rice biofertilization in 1992, and became naturalized in stagnant water (Yanni 1992). It had the highest value of the total nutrients in their living parts (El-Komi, 2002). It forms pure populations especially in the area of control structures and pumping stations (Serag and Khedr, 1996). This species shows abundant growth that is capable of excluding the native Lemna species, particularly in summer (Boulos, 2009) It was recorded, recently, in the northern parts of the Nile Delta (El Saadawi 2000). Vossia cuspidata, another invasive species in the study area, grows in dense and conspicuous populations along Lake Mariut. Its distribution was reported throughout tropical Africa and south-east Asia (Skerman and Riveros, 1989), Migahid (as quoted by Shehata, 1996) reported it as one of the "Sudd plants" in southern Sudan. Symphyotrichum squamatum, as a common species in Omayed site and Lake Mariut, was introduced from Latin America. Its first record in Egypt dates back to the early 1970s. Now it is completely naturalized and is considered as one of the most widespread weeds in Egypt (Boulos and El-Hadidi, 1994; El-Fahar and Sheded, 2002; Shaltout and Al-Sodany, 2002; Shaltout and Galal, 2006; Seif El-Nasr and Bidak, 2006). Eichhornia crassipes (water hyacinth), was introduced to Egypt as an ornamental plant during the rule of Khedive Tawfik (1879-1892); hence it has, for many years, been grown to a limited extent in certain public and private gardens of Cairo and Alexandria (Zahran and Willis, 2003). Now it is the most worst aquatic naturalized weed in Egypt that infests all water courses with large cover and causes major problems such as restricting water flow, transpiring high amounts of water and providing many vectors of human diseases (Shaltout and Galal, 2006). Simpson (1932) reported that it was widely distributed in the fresh water 
channels of the Nile Delta, near Cairo, Alexandria and the brackish water of the northern lakes (e.g. Mariut), however it is killed by sea water

Many of the recorded species in the present study were common segetal and ruderal weeds in the Nile Delta (see Shaltout et al. 2010). For example Chenopodium murale, Convolvulus arvensis, Corchorus olitorius, Cynodon dactylon, Cyperus rotundus, Eruca sativa, Euphorbia helioscopia, Hibiscus trionum, Malva parviflora, Polypogon monspeliensis, Portulaca oleracea, Sonchus asper and Sonchus oleraceus were segetals and Calligonum polygonoides subsp. comosum, Persicaria salicifolium, Polygonum equisetiforme, Salsola kali, Lotus glaber, sesbania sesban, Cynanchum acutum, Phyla nodiflora, Datura innoxia, Plantago lagopus, Silybum marianum, Pluchea discorides, Panicum repens, Dichanthium annulatum and Cyperus laevigatus were ruderals, due to the change of the land use in the study area (e.g. building of tourist resorts and gardens around them), lead to the appearance of a sporadic weed flora, while most of the species of the original plant cover disappear. Before the first weeding, a lot of desert species were still in the gardens, after continuing the human impact (e.g. cleaning, ploughing, etc.) many of them were completely eliminated. In contrast to typical weeds, desert species lack the possibility to persist after this new land use system. The transported soil from the agriculture land of the Nile Delta to raise gardens acted as a seed bank of weeds, and this phenomenon is not restricted to the study area only, but extend to different area as in Red Sea area (Sheded and Shaltout, 1998).

\section{References}

Abdel-Khalik, K.N., 2002. Biosystematic studies on Brassicaceae (Cruciferae) in Egypt. Ph. D. Thesis, Wageningen Univ. 278 pp.

Ahmed, D.A., 2009. Current situation of the flora and vegetation of the western Mediterranean desert of Egypt. Ph. D. Thesis, Tanta Univ., Tanta. 424 pp.

Al-Sodany, Y.M., 1992. Vegetation analysis of the northern part of Nile Delta region. M. Sc. Thesis, Tanta Univ., Tanta. $122 \mathrm{pp}$.

Al-Sodany, Y.M., 1998. Vegetation analysis of the canals, drains and lakes of the northern part of Nile Delta. Ph. D. Thesis, Tanta Univ., Tanta. pp. 232. 
Ammar, M. Y.(1970). An analytical study of the effect of microenvironmental variations on the distribution of vegetation of rocky ridges at Burg El-Arab. M. Sc. Thesis, Alexandria University, Alexandria. pp. 151.

Archibold, O.W., 1995. Ecology of world vegetation. Chapman and Hall, London. 510 pp..

Ayyad, M.A., 1983. Some aspects of land transformation in the Western Mediterranean Desert of Egypt. Adv. Space Res. 8 (2): 19 - 29

Ayyad, M.A., El-Ghareeb, R., 1982. Salt marsh vegetation of the Western Mediterranean desert of Egypt. Vegetatio 49: 3- 19.

Batanouny K.H., 1999. Wild medicinal plants in Egypt. Palm Press, Egypt. $207 \mathrm{pp}$.

Boulos, L., 1997. Endemic flora of the Middle East and North Africa. In Barakat, H. N. and Hegazy, A. K. (eds.): reviews in ecology: Desert Conservation and Development. Metropole, Cairo. 229-260 pp..

Boulos, L., 1999. Flora of Egypt: volume one (Azollaceae - Oxalidaceae). Al-Hadara Publishing, Cairo. 419 pp.

Boulos, L., 2000. Flora of Egypt: volume two (Geraniaceae Boraginaceae). Al-Hadara Publishing, Cairo. 352 pp.

Boulos, L., 2002. Flora of Egypt: volume three (Verbenaceae Compositae). Al-Hadara Publishing, Cairo. 373 pp.

Boulos, L., 2005. Flora of Egypt: volume four Monocotyledons (Alismataceae - Orchidaceae). Al-Hadara Publishing, Cairo. 617 pp.

Boulos, L., 2009. Flora of Egypt checklist, revised annotated edition. AlHadara Publishing, Cairo. 410 pp.

Boulos, L., El-Hadidi, N.M., 1994. The weed flora of Egypt. American University in Cairo Press, Cairo. 361 pp.

Chitek, J., Slavik, B., 1993. Contribution to the flora of Cyprus. 2. Fl. Medit. 3: 239 - 259 .

Cope, T.A., Hosni, H.A., 1991. A key to Egyptian grasses. Royal Botanic Gardens, Kew. 75 pp. 
Crawley, M.J., Harvey, P.H., Purvis, A., 1996. Comparative ecology of the native and alien floras of the British Isles. Philo. Trans. of the Royal Soc. of London 351: 1251 - 1259.

Dallman, P.R., 1998. Plant life in the world's Mediterranean climates. University of California Press, Berkelcy. 257 pp.

Drar, M., 1952. A report on Kochia indica wight in Egypt. Des. Inst. Bull. 2: $54-58$.

El-Bayyoumy, M.A., 1976. Ecological phytosociological studies on the sand dune vegetation at Mariut area. M. Sc. Thesis, Tanta University, Tanta. pp. 83.

El-Demerdash, M.A., 1984. Ecological studies on Juncus plants. Ph. D. Thesis, Mansoura Univ., Mansoura . 156 pp.

El-Demerdash, M.A., Ayyad, M.A., Shaltout, K.H., 1987. Correlation of the distribution of Juncus spp. with the prevalling environmental factors in Egypt. Bull. Fac. Sci., Mansoura Univ. 14 (2): 129 - 156

El-Fahar, R.A., 1989. A phytosociological study on weed vegetation in the Nile Delta region. M. Sc. Thesis, Tanta Univ., Tanta. 128 pp.

El-Fahar, R.A., Sheded, M.G., 2002. Weed flora in plantations of recently established tourist resorts in the Western Mediterranean Coast of Egypt. Egy. J. Biotech. 11: $330-343$.

El-Ghareeb, R.M., 1975. A study of the vegetation - environmental complex of saline and marshy habitats on the northwestern coast of Egypt. Ph.D. Thesis, Alexandria University, Alexandria. pp. 199.

El-Hadidi, M.N., 1980. Flora of Egypt. Taeckholmia Add. Ser. 1: 1-12.

El-Hadidi, M.N., 1993. Natural Vegetation. In Craig, G. M. (ed.): The Agriculture of Egypt. Oxford University Press. pp. 39-62.

El-Hadidi, M.N., (ed.) 2000. Flora Aegyptiaca: volume I, (1,2). Palm Press. Cairo. 321 pp.

El-Hadidi, M.N., Ayyad, M.A., 1975. Floristic and ecological features of Wadi Habis (Egypt). Coll. Intern. du C.N.R.S. 235: 247 - 258

El-Hadidi, M.N., Fayed, A., 1994/955. Material for excursion flora of Egypt (EFE). Taeckholmia 15: 1 - 233. 
El-Hadidi, M.N., Kosinová, J., 1971. Studies on the weed flora of cultivated land in Egypt: preliminary survey. Mitt. Bot. Staat. Münch., 10: 354 - 367.

El-Kady, H.F., 1987. A study of range ecosystems of the Western Mediterranean Coastal Desert of Egypt. Ph.D. Thesis, Tanta University, Tanta. pp. 136.

El-Kady, H.F., Ayyad, M.A., Bornkamm, R., 1995. Vegetation and recent land use history in the desert of Maktala, Egypt. Geo Ecol. 28: 109123.

El-Komi, T.M., 2002. Evaluation of the range plants along the water courses in the Nile Delta. Ph. D. Thesis, Tanta Univ., Tanta. 163 pp.

El Saadawi, W., 2000. Azollaceae in El-Hadidi, M. N.(ed.) Flora aegyptiaca vol.1(2) :41-45. Palm Press, Cairo.

Fahmy, A.G., 1997. Evaluation of the weed flora of Egypt from Predynastic to Graeco-Roman times. Veg. Hist. Archaeobot. 6: 241 247.

Fakhry, A.M., 1994. Species richness and diversity in the vegetation of the Western Mediterranean coastal desert of Egypt. Ph.D. Thesis, Alexandria University, Alexandria. pp. 128.

FAO., 1970. Pre-investment survey of the north-western coastal region; comprehensive account of the project. Technical report ESE: SF / UAR. 49: 109 pp.

Feinbrun-Dothan, N., 1978. Flora Palaestina: part 3 (Ericaceae Compositae). The Israel Academy of Science and Humanities, Jerusalem Academic Press, Jerusalem. 481 pp.

Feinbrun-Dothan, N., 1986. Flora Palaestina: part 4 (Alismataceae Orchidaceae). The Israel Academy of Science and Humanities, Jerusalem Academic Press, Jerusalem. 462 pp.

Grime, J.P., 1974. Vegetation classification by reference to strategies. Nature 250: 26 - 31 .

Hassib, M., 1951. Distribution of plant communities in Egypt. Bull.Fac. Sci., Fouad I Univ. 29: 59-261. 
Hawksworth, D.L., 1995. Biodiversity: measurement and estimation. Chapman and Hall, London. 140 pp.

Hejny, S., Kosinová, J., 1977. Contribution to synanthropic vegetation of Cairo. Publ. Cairo Univ. Herb. 7 \& 8: 273 - 286.

Heneidy, S.Z., 1991. An Ecological Study of the Grazing Systems of Mariut. Egypt. Ph.D. Thesis, Faculty of Science, Alexandria University, Alexandria. pp. 152.

Heneidy, S.Z., Bidak, L.M., 2001. Multipurpose plant species in Bisha, Asir region, south western Saudi Arabia. Journal of King Saud University 13 (Science: $1 \& 2$ ): $11-26$.

Hill, M.O., 1979a. DECORANA - A FORTRAN program for detrended correspondence analysis and reciprocal averaging. Cornell University, Ithaca N.Y. 90 pp.

Hill, M.O., 1979b. TWINSPAN - A FORTRAN program for arranging multivariate data in an ordered two-way table by classification of the individuals and attributes. Cornell University, Ithaca. N.Y. 52 pp.

Hilmy, S.H., 1971. An ecological study on Asphodelus microcarpus Viv. M. Sc. Thesis, Alexandria University, Alexandria. pp. 151.

Judith, H.H., Dawn, R.B., 2003. Ecology and control of introduced plants. Cambridge University Press, 313 pp.

Kamal, S.A., 1988. A study of vegetation and land-use in the western Mediterranean desert of Egypt. Ph. D. Thesis, the Faculty of Science Alexandria University. pp. 194.

Kassas, M., 1955. Rainfall and vegetation belts in arid North-East Africa. Proceedings of Montpellier Symposium: Plant Ecology. UNESCO, Paris. pp. 49-59.

Kassas, M., 1979. Mareotis: past and future. In: Analysis and Management of Mediterranean Desert Ecosystem, Proc. Inter. Workshop of SAMDENE Project, Alexandria Univ.: 24-28.

Khedr, A., Cadotte, M.W., El-Keblawy, A., Loveti-Doust, J., 2002. Phylogenetic diversity and ecological features in the Egyptian flora. Biodiv. Cons. 11: 1809 - 1824. 
Kosinová, J., 1972. On the weed flora and vegetation in Egypt. Egyp. Bot. Soc., Year book. 2: 1 - 6.

Meikle, R.D., 1977. Flora of Cyprus: vol. 1. The Bentham - Moxon Trust, Royal Botanic Garden, Kew. 832 pp.

Meikle, R.D., 1985. Flora of Cyprus: vol. 2. The Bentham - Moxon Trust, Royal Botanic Garden, Kew. 1969 pp.

Montasir, A.H., Hassib, M., 1956. Manual flora of Egypt. Part I. AinShams University. Imprimerie Misr, Cairo. 615 pp.

Quézel, P., 1978. Analysis of the flora of Mediterranean and Saharan Africa. Ann. Miss. Bot. Gard.65: 479 - 534.

Rabinowitz, D., 1981. Seven forms of rarity. In Synge, H. (Ed.): The Biological aspects of rare plant conservation, John Wiley \& Sons Ltd, London. 205 - 217 pp.

Raunkiaer, C., 1937. Plant life forms. Clarendon, Oxford. 104 pp.

Seif El-Nasr, M., Bidak, L., 2005. Conservation and sustainable use of medicinal plants project: national survey, north western coastal region. First Quarterly Report. Mubarak City for Scientific Reserch and Technology Applications. 178 pp.

Seif El-Nasr, M., Bidak, L., 2006. Conservation and sustainable use of medicinal plants project: national survey, north western coastal region. Vol II. Medicinal plants in the Area. Final Report. Mubarak City for Scientific Reserch and Technology Applications. 178 pp.

Selim. A.A., 1969. Geology of El-Sallum Area, Western Mediterranean Coastal Zone, U. A. R.., Ph. D. Thesis, Alex. University.

Serag, M.M., Khedr, A.A., 1996. The shoriline and aquatic vegetation of El-Salam canal, Egypt. J. of Environ. Sci. 11: 141 - 163.

Shaltout, K.H., 1983. An ecological study of Thymelaea hirsuta (L.) Endl. in Egypt. Ph. D. Thesis, Tanta Univ., Tanta. 165 pp.

Shaltout S.K., 2014. Ecological study on the alien species in the Egyptian flora. M. Sc. Thesis, Tanta Unversity, Tanta. pp. 191. 
Shaltout, K.H., Al-Sodany, Y.M., 2002. Phytoecology of Omayed site. MedWetCoast Project, Egyptian Environmental Affairs Agency, Cairo. 89 pp.

Shaltout, K.H., El-Beheiry, M.A., 2000. Demography of Bassia indica in the Nile Delta region, Egypt. Flora 195: 392 - 397.

Shaltout, K.H., El-Ghareeb, R., 1992. Diversity of the salt marsh plant communities in the western Mediterranean region of Egypt. J. Univ. Kuwait (Sci.) 19: 75 - 83 .

Shaltout, K.H., Galal, T.M., 2006. Comparative study on the plant diversity of the Egyptian Northern lakes. J. of Aqua. Res., 32(2): 254270

Shaltout, K.H., Sharaf El-Din, A., Ahmed, D.A., 2010. Plant life in the Nile Delta. Tanta University Press, Tanta, Egypt. 232 pp.

Sheded M.G., Shaltout, K.H., 1998. Weed flora in plantations of recently established tourist resorts along Red Sea coast - Egypt. J. Union Arab Biol. 5 (B): 109 - 119.

Shehata, M.N., 1996. Ecological studies on Vossia cuspidata (Roxb.) Griff. in the Nile Delta of Egypt. Egy. J. of Bot. 36 (1): 37 - 51.

Simpson, N.D., 1932. A report on the weed flora of the irrigation channels in Egypt. Ministry of Public Works, Government Press, Cairo , 124 pp.

Skerman, P.J., Riveros, F., 1989. Tropical grasses. FAO Plant Production and Protection Series No. 23. 749 pp.

Snogerup, S., Snogerup, B., 1993. Additions to the flora of Samos, Greece. Fl. Med. 3: 211 - 222

Täckholm, V., 1956. Students' flora of Egypt. Anglo-Egyption Bookshop, Cairo. 649 pp.

Täckholm, V., 1974. Students' flora of Egypt:(2 $2^{\text {nd }}$ ed.). Cairo University, Cairo. 888 pp.

Täckholm, V., Boulos, L., 1974. Supplementary notes to studients' flora of Egypt. $2^{\text {nd }}$ ed. Publ. of Cairo Univ. Herb. 5:1-135. 
Täckholm, V., Drar, M., 1941. Flora of Egypt: vol. 1. Bull. Fac. Sci., Fuoad I Univ., 17: 3- 574.

Täckholm, V., Drar, M., 1950. Flora of Egypt: vol. 2, Bull. Fac. Sci., Fuoad I Univ., 28: 1- 547.

Täckholm, V., Drar, M., 1954. Flora of Egypt: vol. 3, Bull. Fac. Sci., Cairo Univ., 30: 1- 644.

Täckholm, V., Drar, M., 1969. Flora of Egypt: vol. 4, Bull. Fac. Sci., Cairo Univ., : 1-36.

Tutin, T.G., Heywood, V.H., Burges, N.A., Valentine, D.H., Wealters, S.M., Webb, D.A., Ball, P.W., Chater, A.O., 1964(eds.). Flora Europaea: volume one (Lycopodiaceae to Plantanaceae). University Press, Cambridge. 464 pp.

Tutin, T.G., Heywood, V.H., Burges, N.A., Valentine, D.H., Wealters, S.M., Webb, D.A., Ball, P.W., Chater, A.O., Ferguson, I.K., Moore, D.M., 1968 (eds.). Flora Europaea: vol. 2 (Rosaceae to Umbelliferae). University Press, Cambridge. 455 pp.

Tutin, T.G., Heywood, V.H., Burges, N.A., Valentine, D.H., Wealters, S.M., Webb, D.A., Ball, P.W., Chater, A.O., Ferguson, I.K., Moore, D.M., Defillipps, R.A., Richardson, I.B.K., 1972 (eds.) Flora Europaea: vol. 3 (Diapensiaceae to Myoporaceae). Cambridge University Press, Cambridge. 385 pp.

Tutin, T.G., Heywood, V.H., Burges, I.K., Moore, N.A., Valentine, D.H., Wealters, S.M., Webb, P.W., Chater, D.M., Defillipps, R.A., Richardson, I.B.K., 1976(eds.). Flora Europaea: vol. 4 (Plantaginaceae to Compositae). Cambridge University Press, Cambridge. 505 pp.

UNESCO, 1977. Map of the world distribution of arid regions. MAB Technical Notes, 7.

Waisel, Y., 1972. Biology of halophytes. Academic Press, New York. pp. 395.

Weber, E., 1997. The alien flora of Europe: a taxonomic and biogeographic Review. J. Veg. Sci. 8: 565-572. 
Wickens, G.E., 1976. The Flora of Jebel Marra (Sudan Republic) and its geographical affinities. Kew Bull. Add. Ser. 5. 198 pp..

Yanni, Y.G., 1992. Contribution of inoculation with Azolla combined with nitrogen, phosphorus and zinc to rice in Nile Delta. World J. of Microbiol. Biotech.. 8: 579 - 584.

Zahran, M.A., Willis, A.J., 2003. Plant life in the River Nile in Egypt. Mars Publishing House, Riyadh. pp. 531.

Zahran, M.A., Willis, A.J., 2009. The vegetation of Egypt. $\left(2^{\text {nd }}\right.$ ed.) Springer Netherlands $437 \mathrm{pp}$.

Zohary. M., 1966. Flora Palaestina: part 1 (Equisetaceae - Moringaceae). The Israel Academy of Science and Humanities, Jerusalem Academic Press, Jerusalem. 346 pp.

Zohary. M., 1972. Flora Palaestina: part 2 (Platanaceae - Umbelliferae). The Israel Academy of Science and Humanities, Jerusalem Academic Press, Jerusalem. 489 pp.

Zohary. M., 1973. Geobotanical foundations of Middle East. Gustav Fischer Verlag, Stuttgart. pp. 739. 
Appendix. Presence percentage of characteristic species of the 13 habitats, which identified in the Western Mediterranean region resulted after TWINSPAN classification. CD: Coastal dunes, SF: Sand formation, SP: Sallum plateau, SM: Salt marshes, SD: Saline depressions, NS: Non-saline depressions, IR: Inland ridges, IP: Inland plateaus, WA: Wadis, CL: Cultivated lands, RO: Road sides, SR: Summer resorts, LA: Lake Mariut.

\begin{tabular}{|c|c|c|c|c|c|c|c|c|c|c|c|c|c|}
\hline \multirow[b]{2}{*}{ Species } & \multicolumn{13}{|c|}{ Habitats } \\
\hline & CD & SF & SP & SM & SD & NS & IR & $\mathrm{IP}$ & WA & $\mathrm{CL}$ & RO & SR & LA \\
\hline Achillea santolina $\mathrm{L}$. & & & & & & & & & & 85.5 & & & \\
\hline Aegilops kotschyi Boiss. & & & & & & & & 60 & & & & & \\
\hline $\begin{array}{l}\text { Aeluropus lagopoides (L.) Trin. ex } \\
\text { Thwaites }\end{array}$ & & & & 33.3 & & & & & & & & & \\
\hline Ammophila arenaria (L.) Link & 100 & & & & & & & & & & & & \\
\hline $\begin{array}{l}\text { Anabasis articulata (Forssk.) Moq. } \\
\text { Anagallis arvensis L. }\end{array}$ & & & 100 & 85.5 & & 66.6 & & 60 & & 100 & & & \\
\hline Arisarum vulgare Targ. Tozz. & & & & & & & & & 40 & 75 & & & \\
\hline Artemisia monosperma Delile & & & & & & 75 & & 60 & & & & & \\
\hline $\begin{array}{l}\text { Arthrocnemum macrostachyum } \\
\text { (Moric.) K. Koch }\end{array}$ & & & & 100 & 66.6 & & & & & & & & \\
\hline $\begin{array}{l}\text { Arundo donax } \mathrm{L} \text {. } \\
\text { Asphodelus aestivus Brot. }\end{array}$ & & 60.5 & & 100 & & 100 & 100 & 100 & & & & & 33.3 \\
\hline $\begin{array}{l}\text { Asphodelus aestivus Brot. } \\
\text { Atriplex halimus L. }\end{array}$ & & & 95 & 100 & & & & & & & & & 94.4 \\
\hline Atriplex portulacoides $\mathrm{L}$. & & & & 75 & & & & & & & & & \\
\hline $\begin{array}{l}\text { Atriplex semibaccata } \mathrm{R} . \mathrm{Br} \text {. } \\
\text { Avena fatua } \mathrm{L} \text {. }\end{array}$ & & & & & & & & & & 666 & & & 94.4 \\
\hline Azolla filiculoides Lam. & & & & & & & & & & 00.0 & & & 83.3 \\
\hline Bassia indica (Wight) A. J. Scott & & & & & & & & & & & & & 66.6 \\
\hline $\begin{array}{l}\text { Beta vulgaris L. subsp. maritima } \\
\text { (L.) Arcang. }\end{array}$ & & & & & & & & 80 & & & & & \\
\hline Calendula arvensis $\mathrm{L}$. & & & & & & & & & 40 & & & & \\
\hline $\begin{array}{l}\text { Capparis spinosa } \text { L. var. inermis } \\
\text { Turra }\end{array}$ & & & & & & & & & 56 & & & & \\
\hline $\begin{array}{l}\text { Carthamus glaucus M. Bieb.subsp. } \\
\text { alexandrinus (Boiss. \& Heldr.) } \\
\text { Hanelt }\end{array}$ & & & 95 & & & & & & & & & & \\
\hline Ceratophyllum demersum $\mathrm{L}$. & & & & & & & & & & & & & 50 \\
\hline Chenopodium album $\mathrm{L}$. & & & & & & & & & & 75 & & & \\
\hline Convolvulus arvensis $\mathrm{L}$. & & & & & & & & & & 66.6 & & & \\
\hline Cressa cretica $\mathrm{L}$. & & & & 75 & & & & & & & & & \\
\hline Crucianella maritima $\mathrm{L}$. & 50 & & & & & & & & & & & & \\
\hline $\begin{array}{l}\text { Cutandia dichotoma (Forssk.) } \\
\text { Batt. \& Trab. }\end{array}$ & & & & & & & & & & & & & 83.3 \\
\hline Cynanchum acutum $\mathrm{L}$. & & & & & & & & & & & & & 100 \\
\hline Cynodon dactylon (L.) Pers. & & & & & & & & & & & & 100 & \\
\hline Dactylis glomerata $\mathrm{L}$. & & & & & & & 66.6 & & & & & & \\
\hline Deverra tortuosus (Desf.) DC. & 100 & 40 & 100 & & & & 66.6 & & & & 96 & & \\
\hline $\begin{array}{l}\text { Echinochloa stagnina (Retz.) P. } \\
\text { Beauv. } \\
\text { Echinops spinosus L. }\end{array}$ & 40.5 & & & & & & 100 & & & & & & 100 \\
\hline
\end{tabular}


Appendix. Cont. 1.

\begin{tabular}{|c|c|c|c|c|c|c|c|c|c|c|c|c|c|}
\hline \multirow[t]{2}{*}{ Species } & \multicolumn{13}{|c|}{ Habitats } \\
\hline & $\mathrm{CD}$ & SF & SP & SM & SD & NS & IR & IP & WA & $\mathrm{CL}$ & RO & SR & LA \\
\hline Echiochilon fruticosum Desf. & 33.3 & & & & & 66.6 & & & & & & & \\
\hline $\begin{array}{l}\text { Eichhornia crassipes (C. Mart.) } \\
\text { Solms }\end{array}$ & & & & & & & & & & & & & 100 \\
\hline Eryngium campestre $\mathrm{L}$. & & & & & & & & & & 85.5 & & & \\
\hline Euphorbia paralias Aiton & 33.3 & & & & & & & & & & & & \\
\hline Glebionis coronarium (L) Tzvelev & & & & & & & & & & 100 & & & \\
\hline Globularia arabica Jaub. \& Spach. & & & & & & & 100 & & & & & & \\
\hline $\begin{array}{l}\text { Halocnemum strobilaceam (Pall.) } \\
\text { M. Bieb. }\end{array}$ & & & & 85.5 & 66.6 & & & & & & & & 100 \\
\hline $\begin{array}{l}\text { Haloxylon salicornicum (Moq.) } \\
\text { Bunge ex Boiss. }\end{array}$ & & 40 & 100 & & & & & & & & & & \\
\hline Helianthemum lippii (L.) Dum. & & & & & & & 805 & 60 & & & & & \\
\hline $\begin{array}{l}\text { Herniarla hirsuta } \mathrm{L} . \\
\text { Launaea nudicaulis }\end{array}$ & & & & & & & 89.5 & & & & & & 100 \\
\hline $\begin{array}{l}\text { Launeae fragilis (Asso) Pau subsp. } \\
\text { fragilis }\end{array}$ & 66.5 & & & & & & 66.6 & & & 85.5 & & & \\
\hline Limbarda crithmoides (L.) Dumart. & & & & & & & & & & & & & 60 \\
\hline Limoniastrum monopetalum (L.) & & & & 100 & 100 & & & & & & & & \\
\hline Boiss. & & & & & & & & & & & & 666 & \\
\hline Lolium perenne $\mathrm{L}$. & & & & & & & & & & & & 60.6 & \\
\hline $\begin{array}{l}\text { Ludwigia stolonifera (Guill. \& } \\
\text { Perr.) P. H. Raven }\end{array}$ & & & & & & & & & & & & & 57.1 \\
\hline Lycium shawii Roem. \& Schult. & & & & & & & & 60 & & & & & \\
\hline Lythrum junceum Banks \& Sol. & 85.5 & & & & & & & & & & & & \\
\hline Malva parviflora $\mathrm{L}$. & & & & & & & & 80 & & & & 93.1 & 66.6 \\
\hline Medicago polymorpha $\mathrm{L}$. & & & & & & & & & & 75 & & & \\
\hline Myriophyllum spicatum L. & & & & & & & & & & & & & 66.6 \\
\hline $\begin{array}{l}\text { Najas marina L. subsp. armata }(\mathrm{H} \text {. } \\
\text { lindb.) Horn }\end{array}$ & & & & & & & & & & & & & 100 \\
\hline $\begin{array}{l}\text { Noaea mucronata (Forssk.) Asch. } \\
\text { \& Schweinf. }\end{array}$ & & 40 & & & & & 66.6 & & & & & & \\
\hline Ononis vaginalis Vahl & 33.3 & & & & & & & & & & & & \\
\hline Onopordum alexandrinum Boiss. & & & & & & & & & & 75 & & & \\
\hline Phlomis floccosa D. Don & & & & & & & & & 33.3 & & & & \\
\hline $\begin{array}{l}\text { Phragmites australis (Cav.) Trin. } \\
\text { ex Steud. subsp. australis }\end{array}$ & & & & & & & & & & & & & 100 \\
\hline Plantago albicans L. & & & & 33.3 & & 100 & 75 & & & & & & \\
\hline Pluchea discorides (L.) DC. & & & & & & & & & & & & & 60 \\
\hline Polygonum equisetiforme $\mathrm{Sm}$. & & & & & & & & & & & 85.5 & & \\
\hline Polypogon monspelensis (L.) Desf. & & & & & & & & & & & & 96.6 & \\
\hline Potamogeton pectinatus $\mathrm{L}$. & & & & & & & & & & & & & 100 \\
\hline Reichardia picroides (L.) Roth. & & & & & & & & & & & & & 66.6 \\
\hline Salsola tetrandra Forssk. & & & & 33.3 & 100 & & & & & & & & \\
\hline Salvia lanigera Poir. & & & & & & & 66.6 & & & & & & \\
\hline $\begin{array}{l}\text { Sarcocornia fruticosa (L.) A. J. } \\
\text { Scott }\end{array}$ & & & & 100 & & & & & & & & & 100 \\
\hline
\end{tabular}


K. H. Shaltout et al.

Appendix. Cont. 2

\begin{tabular}{|c|c|c|c|c|c|c|c|c|c|c|c|c|c|}
\hline \multirow{2}{*}{ Species } & \multicolumn{13}{|c|}{ Habitats } \\
\hline & \begin{tabular}{|l|}
$C D$ \\
\end{tabular} & SF & SP & SM & SD & NS & IR & IP & WA & $\mathrm{CL}$ & RO & SR & LA \\
\hline Sarcocornia fruticosa (L.) A. J. & & & & 100 & & & & & & & & & 100 \\
\hline Scorzonera undulata Vahl & & & & & 66.6 & & 66.6 & & & & & & \\
\hline $\begin{array}{l}\text { Senecio glaucus L. subsp. } \\
\text { coronopifolium (Maire) C. Alexande }\end{array}$ & & & & & & & & & & & & & 66.6 \\
\hline Senecio glaucus. L. subsp. glaucus & & & & & & & & & & & & & 55.5 \\
\hline $\begin{array}{l}\text { Silybum marianum } \\
\text { var. } \text { L.) Gaertorum. }\end{array}$ & & & & & & & & & & & 49 & & \\
\hline Sinapis allionii Jacq. & & & & & & & & & & & & & 100 \\
\hline Sonchus oleraceus $\mathrm{L}$. & & & & & & & & & & & & 93.1 & 100 \\
\hline $\begin{array}{l}\text { Stipagrostis ciliata (Desf.) De } \\
\text { Winter }\end{array}$ & & & & & & & & 60 & & & & & \\
\hline Suaeda monoica Forssk. & & & & 66.6 & & & & & & & & & \\
\hline Suaeda pruinosa Lange & & & & & 66.6 & & & & & & & & \\
\hline Tamarix nilotica (Ehrenb.) Bunge & & & & & & & & & & & & & 94.4 \\
\hline $\begin{array}{l}\text { Thymelaea hirsuta (L.) Endl. } \\
\text { Thymus capitatus (L.) Link }\end{array}$ & 33.3 & 66.7 & 100 & 66.6 & & 66.6 & $\begin{array}{c}75 \\
100\end{array}$ & 60 & 75 & & 43 & & \\
\hline $\begin{array}{l}\text { Typha domingensis (Pers.) Poir. ex } \\
\text { Steud. }\end{array}$ & & & & & & & & & & & & & 100 \\
\hline $\begin{array}{l}\text { Volutaria lippii (L.) Cass. ex } \\
\text { Maire }\end{array}$ & & & & & & & & & & & & & 83.3 \\
\hline $\begin{array}{l}\text { Zilla spinosa ( L.) Prantl subsp. } \\
\text { biparmata (O. E. Schulz) Maire \& } \\
\text { Weiller }\end{array}$ & & & & & & & & & 33.3 & & & & \\
\hline Zygophyllum album $\mathrm{L}$. & & & & & & 66.6 & & & & & & & \\
\hline
\end{tabular}

